Revue internationale de l'économie sociale

Recma

Charles Gide au $x^{e}$ siècle, Luc Marco (dir.). Comité pour

l'édition des oeuvres de Charles Gide, L'Harmattan, Paris, 2011, $254 \mathrm{p}$.

\title{
Michel Dreyfus
}

Numéro 323, janvier 2012

URI : https://id.erudit.org/iderudit/1018348ar

DOI : https://doi.org/10.7202/1018348ar

Aller au sommaire du numéro

Éditeur(s)

Association Recma

ISSN

1626-1682 (imprimé)

2261-2599 (numérique)

Découvrir la revue

Citer ce compte rendu

Dreyfus, M. (2012). Compte rendu de [Charles Gide au xx ${ }^{e}$ siècle, Luc Marco (dir.). Comité pour l'édition des oeuvres de Charles Gide, L’Harmattan, Paris, 2011, 254 p.] Revue internationale de l'économie sociale, (323), 113-114. https://doi.org/10.7202/1018348ar d'utilisation que vous pouvez consulter en ligne.

https://apropos.erudit.org/fr/usagers/politique-dutilisation/ 


\section{NOTES DE LECTURE}

\section{Charles Gide au xIx ${ }^{\mathrm{e}}$ siècle \\ Luc Marco (dir.). Comité pour l'édition \\ des œuvres de Charles Gide, L'Harmattan, \\ Paris, 2011, $254 p$.}

Ce livre se décline autour de deux grandes parties. La première est consacrée à l'examen des conceptions de Charles Gide. Sa pensée reste très actuelle, bien que, comme nous le rappelle Denis Clerc, la " république gidienne ", qui reposait sur la primauté des coopératives de consommation, ne s'est jamais concrétisée; dans la perspective de Charles Gide, la coopération de production ne jouait qu'un rôle subalterne. La prédominance de la coopération de production commença à être remise en question, au lendemain de la mort de Charles Gide, par Georges Fauquet, qui dans son Secteur coopératif (1935) appelait les deux grandes composantes de la coopération à mieux collaborer entre elles. Cet appel, il faut le dire, resta longtemps sans grands résultats. Puis, à partir des années 60, Henri Desroche et Claude Vienney posèrent les bases de ce qui allait devenir, à partir de 1977, l'économie sociale. Une nouvelle inflexion apparut deux décennies plus tard avec l'émergence de l'économie sociale et solidaire (ESS): on ne peut discuter ici des rapports entre cette dernière et l'économie sociale. Pourtant, on le vérifie chaque jour, le libéralisme montre ses effets nocifs sur le plan économique et social. Ce constat rend la pensée de Charles Gide plus vivante que jamais et il est donc justifié de la mettre en perspective, de voir ce qu'elle fut et ce qu'elle peut nous apprendre aujourd'hui.

D'abord, que fut-elle du vivant de Charles Gide, se demandent Albert Broder, Annie Cot et Yves Breton? Tous trois cherchent à définir la place de cet économiste humaniste " au temps de la première mondialisation " que furent les années 1880-1930. Sa tentative de synthèse entre protectionnisme et libre-échange, sa critique de l'école libérale, puis ses interrogations, incessantes durant ce demi-siècle, sur la notion de progrès dans le champ économique sont autant de questions qui se posent de nos jours. Le contexte est évidemment très différent: le rôle de l'Etat dans l'économique et le social a notamment beaucoup changé. Son intervention a considérablement progressé dans l'entre-deux-guerres, puis à la Libération. Cette tendance a semblé s'approfondir encore en 1981, mais quelques années plus tard, une inflexion s'est produite et l'on se demande aujourd'hui jusqu'où ira le désengagement de l'Etat dans l'économique et le social. En un sens, cette tendance est peut-être une chance pour l'économie sociale, dans la mesure où cette inflexion du rôle de l'Etat lui laisse une plus grande place. Mais cette situation ne lui confie-t-elle pas aussi des responsabilités qui excèdent ses forces? La question s'est déjà posée dans le passé, comme le montre notamment l'histoire de la protection sociale: il fallut attendre l'avènement de la Sécurité sociale, à la Libération, pour que soit mis en place un système destiné à l'ensemble de la population du pays. Enfin, comme nous le rappelle Danièle Demoustier, Charles Gide publia lors de l'Exposition universelle de 1900 un ouvrage consacré à l'économie sociale. Lorsque Henri Desroche remit cette notion à l'ordre du jour en 1977 pour définir un principe commun aux coopératives, aux mutuelles et aux associations gestionnaires, il le fit en référence explicite à l'ouvrage de Charles Gide. La notion d'organisme à but non lucratif ne lui semblait en effet pas pertinente pour englober dans un même ensemble des organismes non marchands (associations et mutuelles) et les entreprises marchandes que sont les coopératives. C'est dire combien il faut partir de Charles Gide pour comprendre ce qu'est aujourd'hui l'économie sociale, et peut-être aussi l'ESS. Cela est 
d'autant plus nécessaire que, comme le souligne Danièle Demoustier, la question sociale, que l'on croyait avoir résolue dans le cadre de la croissance fordiste des Trente Glorieuses, est plus que jamais de retour depuis la décennie 80 .

Ce volume s'inscrit aussi dans une histoire éditoriale, puisqu'il résulte notamment de l'action de la Fondation du Crédit coopératif, que Jacques Moreau présida de 1984 à 2003 . Cette fondation et d'autres acteurs de l'économie sociale, notamment la Délégation interministérielleàl'économie sociale (Dies), fondée en 1982, menèrent à bien diverses initiatives pour populariser l'économie sociale, sous la forme d'études, de recherches et de publications. Après avoir réactivé la vieille Revue des études coopératives, fondée en 1921 et que nos lecteurs connaissent aujourd'hui sous la forme de la Recma, ces différents acteurs mirent sur pied à partir de 1994 un Comité pour l'édition des ouvres de Charles Gide. Ce comité fut placé sous la direction scientifique de Marc Pénin, qui écrivit en 1997 une biographie du principal penseur de la coopération de consommation ${ }^{(1)}$. Puis à partir de 1999, le comité publia douze volumes d'écrits de Charles Gide. La seconde partie de cet ouvrage est donc consacrée à l'historiographie gidienne, à travers une étude réalisée par Luc Marco sur la réception dont bénéficia l'Histoire des doctrines économiques depuis les physiocrates jusqu'à nos jours, que Charles Gide et Charles Rist publièrent en 1909, et à travers les deux contributions fournies par André Chomel et Marc Pénin. Nul n'était mieux placé qu'André Chomel pour relater l'histoire, en particulier institutionnelle, du « long silence » dans lequel était tombél'œuvre de Charles Gide depuis des décennies. De son côté, Marc Pénin trace un bilan de la publication de l'œuvre de Charles Gide, de son vivant, puis à partir de 1999. Il explore enfin quelques pistes possibles, d'abord parce que la présente édition ne prétend pas être une publication intégrale, ensuite parce qu'il faudra tenir compte des conditions nouvelles de l'édition et notamment de l'explosion del'édition

(1) Marc Pénin, 2007, Charles Gide (1847-1932), l'esprit critique, L'Harmattan-Comité pour l'édition des oeuvres de Charles Gide. informatique. Une chronologie intellectuelle détaillée de Charles Gide est établie par Germain Hodonou. En conclusion, Thierry Jeantet propose plusieurs objectifs à l'économie sociale. La table des matières des douze volumes publiés par le comité clôt cet ouvrage. Cette seconde partie est également très intéressante. D'une part, elle fournit des éléments très riches sur l'accueil reçu par l'œuvre de Charles Gide en France; il serait d'ailleurs souhaitable de poursuivre cette enquête àl'étranger. D'autre part, à travers le Comité pour l'édition des œuvres de Charles Gide a lieu un moment important de l'histoire de l'économie sociale, qui commença à être reconnue, mais au risque de se voir institutionnalisée, à partir des années 80 .

Cet ouvrage publie les actes d'un colloque récent consacré à celui qui fut longtemps le principal penseur de la coopération. Il met donc un point d'orgue à l'aventure éditoriale entreprise en 1997. Mais si le Comité pour l'édition des œuvres de Charles Gide a décidé de se dissoudre au terme de la mission qu'il s'était fixée, il va être mis prochainement sur pied une Association des amis de Charles Gide. D'autres initiatives sont prises, en France et dans le monde pour populariser et développer l'économie sociale, telles que les Rencontres du Mont-Blanc, qui ont tenu leur cinquième session en novembre 2011. Est-il besoin d'insister longuement pour dire que ces questions concernent au premier chef l'ensemble des composantes de l'économie sociale et de l'ESS, et pas seulement dans notre pays?

Michel Dreyfus

\section{Toward contemporary co-operative studies: Perspectives from Japan's consumer Co-ops \\ The consumer Co-operative Institute of Japan (CCIJ). Tokyo, 2011, 247 p.}

Si l'économie sociale et solidaire (ESS) se distingue par l'universalité de ses principes, ceux-ci ont pu s'incarner sous des formes différentes selon les aires géographiques. Cette diversité 